\title{
Séparation et réparation : expression spatial de l'inachevé au Japon
}

Separation and restoration: spatial expression of uncompleteness in Japan

\section{Hiroshi Kashiwagi \\ Traducteur : Jane Cobbi}

\section{OpenEdition \\ Journals}

\author{
Édition électronique \\ URL : https://journals.openedition.org/tc/5800 \\ DOI : $10.4000 /$ tc. 5800 \\ ISBN : $1952-420 X$ \\ ISSN : 1952-420X \\ Éditeur \\ Éditions de l'EHESS
}

\section{Édition imprimée}

Date de publication : 15 décembre 2011

Pagination : 84-97

ISBN : 978-2-7351-1437-5

ISSN : 0248-6016

Référence électronique

Hiroshi Kashiwagi, «Séparation et réparation : expression spatial de l'inachevé au Japon », Techniques \& Culture [En ligne], 57 | 2011, mis en ligne le 30 juin 2012, consulté le 29 septembre 2022. URL : http:// journals.openedition.org/tc/5800; DOI : https://doi.org/10.4000/tc.5800 


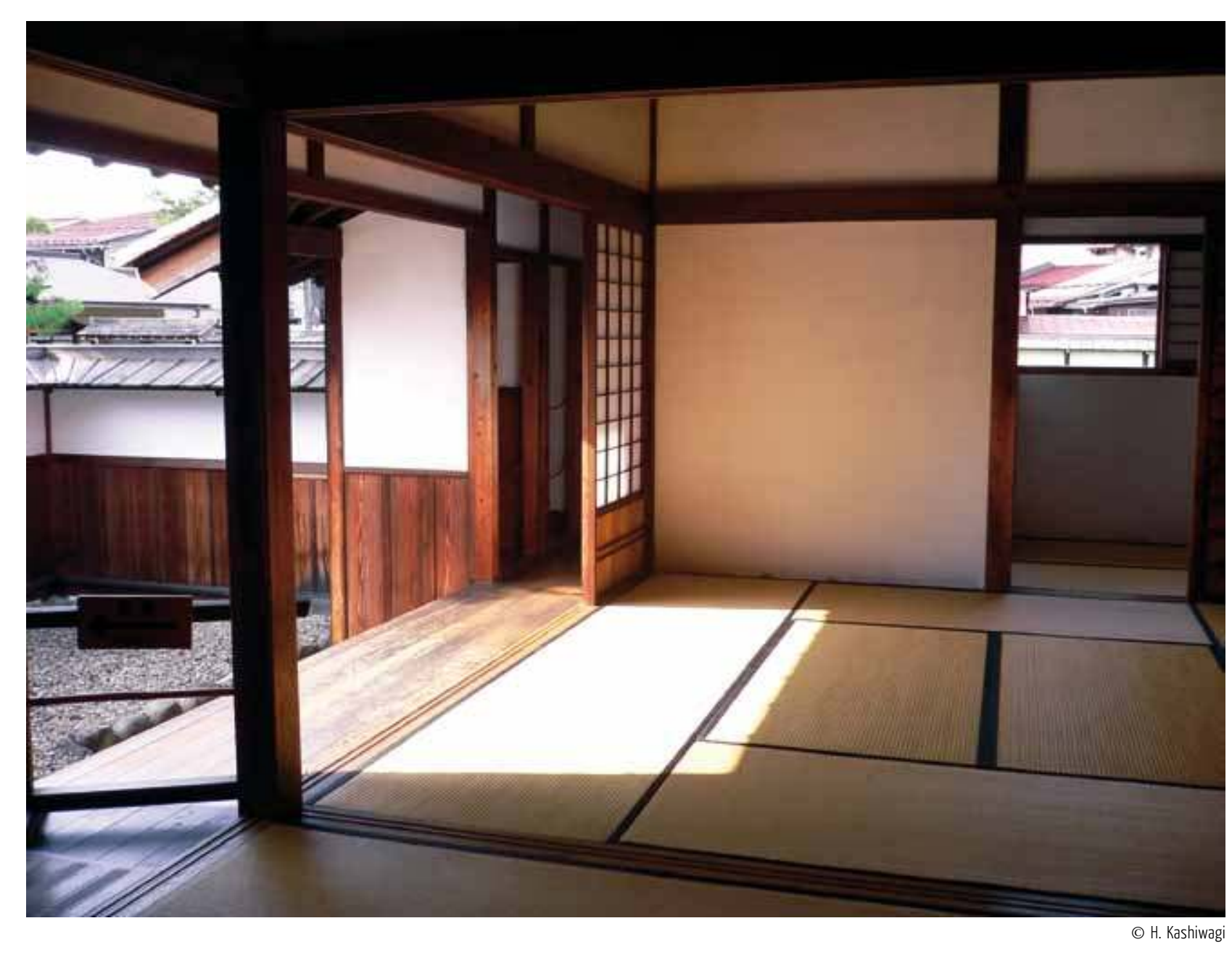

\title{
SÉPARATION ET RÉPARATION
}

Expression spatiale de l'inachevé au Japon

\begin{abstract}
Deux notions japonaises, shikiri et tsukuroi, que l'on peut traduire respectivement par « séparation » et « réparation », donnent accès à un mode de représentation propre à la culture japonaise.

Chacune de ces deux notions permet de saisir une vision, un sentiment, voire un mode de penser particuliers, qui évoquent l'inachèvement, suggèrent la valeur attribuée à l'incomplétude.

En examinant des exemples pris dans différents domaines de la culture japonaise, jaborderai indépendamment ces deux notions exprimées par les termes shikiri et tsukuroi, qui n'ont pas entre elles de relation directe, mais renvoient toutes deux à l'idée d'imperfection.
\end{abstract}

Shikiri

La notion de cloisonnement renvoie au registre du temps comme de l'espace, à la répartition des fonctions spatiales (fonction dévolue à l'espace de la chambre à coucher ou de la salle a manger, par exemple), conme à la division du temps (temps sacre et temps

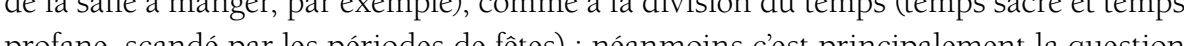

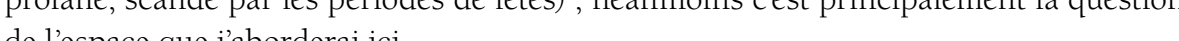
de l'espace que jaborderai ici.

Dans habitation japonaise, le « cloisonnement » traditionnel est extrêmement souple et flexible. Il ne ressemble en rien aux cloisons en dur que sont les murs en occident, et paraitt bien insuffisant, dès lors qu'on le regarde du point de vue de la division de l'espace pratiquée en Europe 
Les habitations traditionnelles du Japon sont faites essentiellement d'un toit posé sur des piliers, avec peu de cloisons fixes. Ce style d'architecture caractérise aujourd'hu encore les sanctuaires shinto. Le toit peut être fait de chaume, de bardeaux ou de tuiles. On ne connaît pas la genèse précise de ce type d'architecture, mais on en trouve une origine dans les habitations de l'époque Yayoi, jusquau III' siècle de notre ère. L'anthropologue Kon Wajiro (1958: 6) a montré l'analogie de ces habitations rurales avec les cabanes à toit de chaume utilisées par les charbonniers dans les provinces japonaises jusqu'au milieu du $x x^{e}$ siècle.

Après le IX ${ }^{e}$ siècle, les bâtiments sont largement ouverts, au moyen de nombreuses cloisons comme les panneaux à claire-voie, qui coupent la perspective, mais laissen passer les sons et la lumière. Ce type de cloison ne constitue pas une séparation nette avec l'extérieur ; de même, les panneaux de tissu et les stores qui divisent les pièces à l'intérieur ne ferment pas les espaces de façon complète, comme le ferait un mur solide. À partir du xue sièce, on utilise fréquemment les paravents, qui perme un 作 certaine A

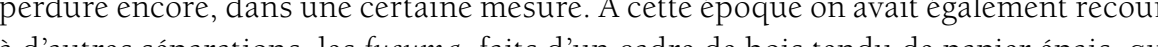
à doutres separations, les fusuma, faits dru cadre de bois tendu de papier epais, que lon deval saisir un a un pour les deplacer. Par la sulte, ces fusuma, dont le non complet est fusuma-shojl, ont eté munis dun dispositif de coulisses qui permet de les faire gisser pour modifier le volume dune piece. En ecartant les fusuma on peut ainsi agrandir lespace, il anive aussi qu'on les retire completement pour former une seule grande pièce. Il s'agit donc de cloisons mobiles, que l'on peut à loisir ouvrir complètement ou partiellement, mais qui, en raison du matériau-papier qui les constitue, ne sont pas étanches. Ces fusuma font l'objet d'aménagements esthétiques variés et de décors multiples : leur grande surface plane se prête à des inventions picturales ou calligraphiques reconnues comme formes artistiques originales dès le xvl siècle (époque Momoyama).

Plus minces que les fusuma, et encore moins solides, les séparations dites shôji, tendues d'une seule couche de papier blanc, sont en usage depuis le xIII' siècle environ. Ces shôji translucides sont dits « akari-shôji »; on apprécie particulièrement la douceur de la lumière tamisée que répand le papier de ces shôji. Le goût pour la lumière diffuse a lui aussi donné rapidement naissance à diverses formes d'agencements et d'inventions esthétiques.

Généralement fermé par des cloisons qui coulissent à l'intérieur de l'habitation, un couloir à plancher de bois longe les pièces principales de la maison. C'est le engawe (littéralement « couloir de liaison »), espace intermédiaire entre le dedans et le dehors du bâtiment. Si on écarte complètement les cloisons mobiles, les pièces qui donnent sur cette galerie-engawa peuvent rester grand ouvertes sur le jardin ou le paysage extérieur. Le cloisonnement japonais ne se limite pas seulement au dispositif offert par le shôji et les fusuma. Il existe aussi des séparations partielles, comme les murs suspendu sous plafond, sagarikabe, et les panneaux-impostes, ranma, qui n’arrêtent pas le regard, bien qu'ils marquent une division de l'espace.

Au-dessus des cloisons mobiles, les panneaux fixes posés en impostes laissent passer lair et la lumiere d une pièce a l'autre. Dans certaines maisons, pour ce panneau-imposte dit ranma, on a tiré parti de l'effet esthétique de planches de bois dites « repas d'insectes» (mushi-gui) : il s'agit de planches sculptées en creux, comme si elles étaient rongées par les xylophages.

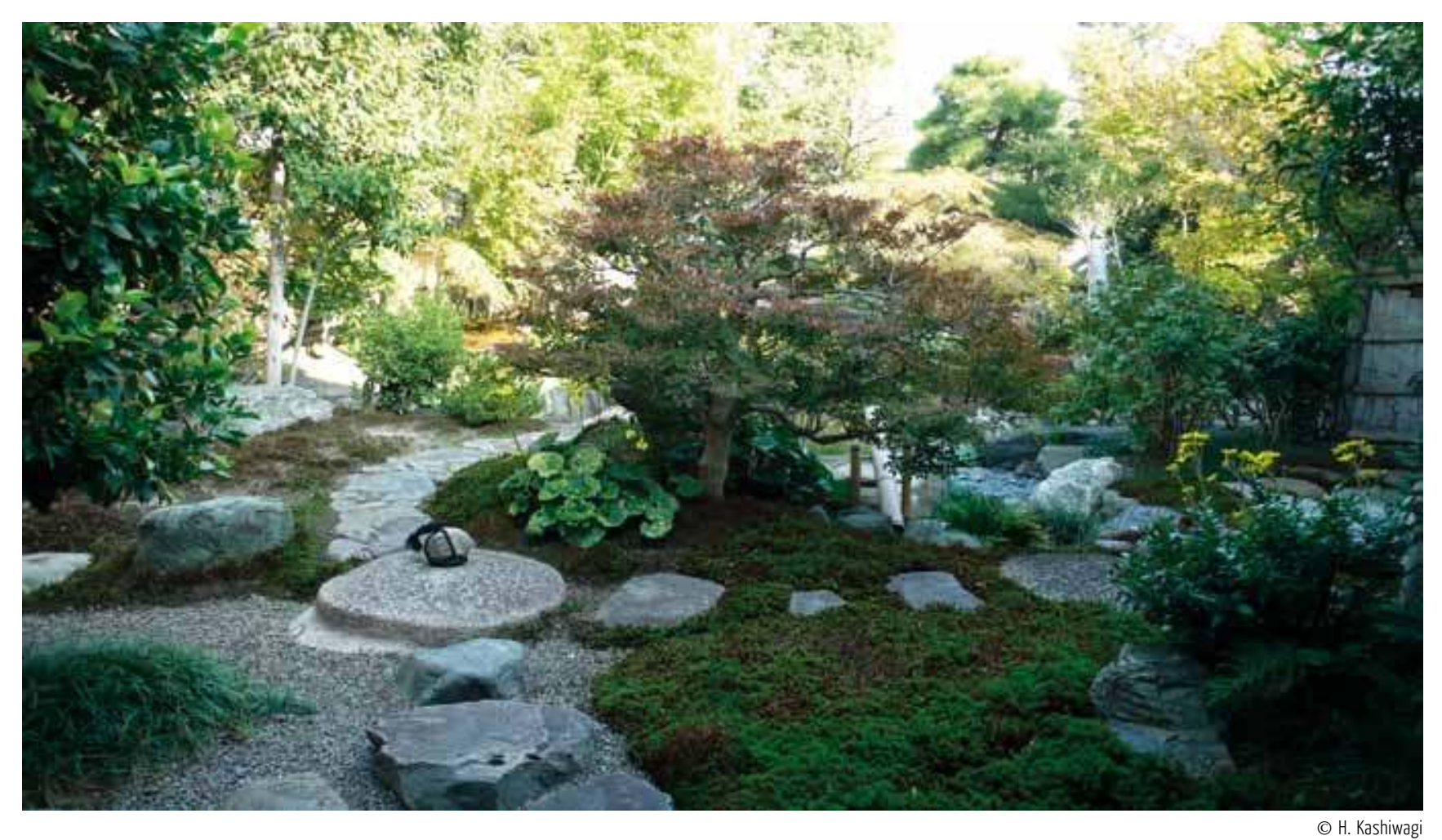

Jardin du temple Kôtồin à Kyoto

appierre nouvé (sekimori-ishi)

ust un moded de séporation qui signifie
bien quion ne doit pas oller au-dela.
Maison de thé

Pierre pour fermer une allée.

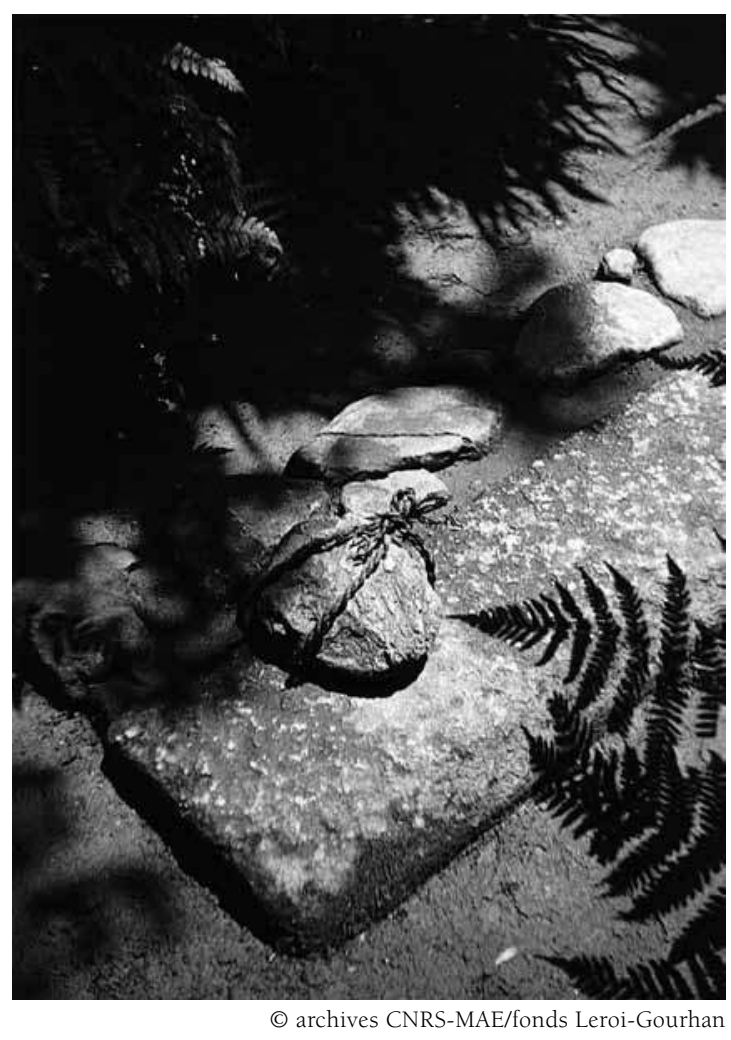




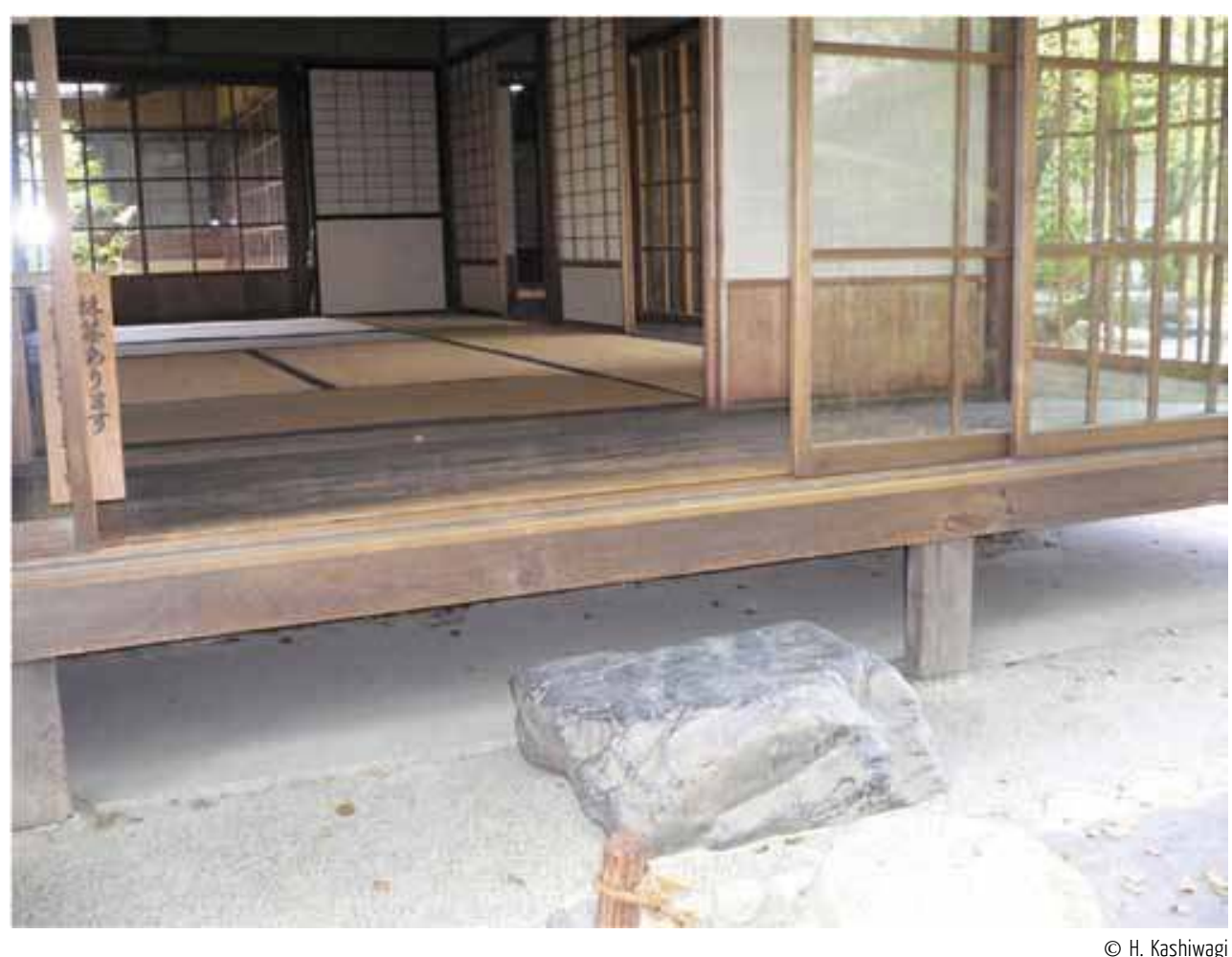

Pour diviser l'espace, on a recours non seulement à des séparations verticales mais auss à des séparations horizontales. Evoquons d'abord les moines bouddhistes qui marchent en portant un parapluie sur le dos, et qui, au moment de faire leur prédication, ouvrent leur portan un pour délimiter un espace sacré, comme dans l'espace intérieur des tomples. Autre paxemple, on voit parfois, duns lesjardins, une pierre altachée par une corde (sechies. Autr-ishi) chargée de delinifer un espace à ne pas franchir Posée sur un he in la piere nouce est chargee de delimiter un espace à pas franchir. Posee sur unchemin, la pierre nouée es destimee à de pas aller au-delà. Notons cependant que beaucoup de jeunes, qui ignorent aujourd'hu cette signification, passent de lautre côte avec désinvolture.

La différence de nature du sol permet aussi de souligner la séparation entre deux espaces : ainsi, dans une même maison, sol couvert d'un plancher et sol recouver de tatami contribuent à créer deux univers différents. Le style d'aménagement es aussi un moyen de marquer la différenciation entre deux pièces : la séparation repose alors sur le recours au style dit «à la japonaise » ou «à l'occidentale ». Ainsi, table et chaises meublent les pièces de style occidental, considérées comme des lieux de réception dans la maison, alors que les pièces à tatami constituent souvent des espaces plus intimes, à usage privé.

L'aménagement intérieur «à l’occidentale », répandu à partir des années 1920 est presque complètement généralisé aujourd'hui ; cependant, la coutume de se déchausser dans l'entrée d'une habitation (ou d'un bâtiment fermé) est restée la même, car l'entrée fait séparation entre l'intérieur et l'extérieur. Un simple dénivellement permet alors la division de l'espace. Dans l'entrée des habitations contemporaines, c'est le niveau du seuil qui marque la séparation entre le dedans et le dehors : on enfile les pantoufles préparées sur la partie surélevée, en laissant ses chaussures en bas. Parfois un panneau de toile, noren, ou de bambou, sudare, vient souligner encore cette séparation

On constate que ce cloisonnement matériel, cette séparation physique obtenue par un dispositif spécifique, se retrouve dans le domaine des relations sociales. Il vaudrait peut-être mieux dire que c'est la façon même dont nous concevons nos relations humaines et nos liens sociaux, qui se manifeste dans la notion de séparation. Dans une expression comme « le seuil est trop haut » (shikii ga takai), ce n'est pas de dénivellement matériel dont il s'agit, mais d'une frontière implicite entre deux niveaux sociaux, dont on a plus ou moins conscience.

Pour aller plus loin, soulignons que les cloisons mobiles que sont les shôji et les fusuma laissent passer les ombres et les bruits, si bien que l'on perçoit le moindre signe de présence de l'autre côté de la cloison. Les portes à panneau plein ou les cloisons à claire-voie, tout en séparant le dedans et le dehors, ou deux espaces intérieurs, permettent aussi de percevoir toute présence, ou d'entendre les bruits et conversations. Néanmoins, dans des situations délicates - où il vaudrait mieux ne pas savoir - celui qui a pu entendre fait comme s'il navait rien entendu, celui qui a pu voir comme s'il n’avait rien vu. Il y a en effet aussi un accord tacite de discrétion. Si comme on le voit, le cloisonnement au Japon permet de se rendre compte de ce qui se passe de l'autre côté, ce n’est pas sans effet sur la conception des rapports sociaux, voire sur la morale sociale.

Quel que soit le type de séparation, il formalise la relation établie entre l'intérieur et l'extérieur. Autrement dit, la notion de séparation reflète ce que l'on considère comme lexterne êr. 1910-1920, apparât au Japon l'idée qu'il faut supprimer le plan ou l dispostion inées 1910-1920, apre reure, dans lequelle la separation incertanne, loue et souple, est marquee par les fusuma. llest alors requle la se séparês chambre privée et espace collectir (du living ou de la salle à manger), a l intérieur du foyer domestique. Notons que c'est à cette période que le Japon a tenté d'adopter la notion de frontière entre privé et public, qui prévalait en Europe.

La séparation entre espace collectif et espace individuel renvoie à une vision moderne de l'opposition public-privé (kô-shi). En Europe, cette bipartition entre public et privé est apparue au xvIII siècle, comme l'indiquent plusieurs historiens, dont Philippe Ariès (1980). A l'intérieur de l'habitation, les murs des chambres séparent les individus qui composent la cellule familiale. La séparation entre le collectif et le privé entraîne la prise de conscience d'une distinction des rapports avec un particulier ou avec le groupe.

Cette séparation entre collectif et privé n’a pas seulement entraîné une prise de conscience de l'individuel et du social, mais aussi, à l'intérieur de la cellule familiale, une prise de conscience de la séparation et de l'indépendance entre les membres et l'ensemble du foyer, ainsi qu'entre chacun des membres d'une famille. La nécessité d'une distinction entre le collectif et l'indivic la période qui va des années 1910 à la fin du xxe siècle.

La séparation entre public et privé en Europe reflète la vision du « social » dans sa conception moderne. Au Japon ce qui prévaut souvent, ce n'est pas la notion de société au sens moderne, mais la notion de seken, de hitosama, ou sotosama. Ces termes peuvent être traduits par : « le monde », « le quartier », « le voisinage », « les gens », et rapprochés de formules comme «ce n'est pas admis », « ce n'est pas acceptable » 
Le mot seken peut renvoyer aussi bien à l'exterieur du foyer domestique, que du groupe social, du village ou de lagglomer sémantique bouge aisément, et dont les frontières ne sont pas fermement fixées, ce qui fait écho au mode de séparation que nous avions vu pour les paravents et les fusuma. De ce point de vue, le fonctionnement des relations sociales au Japon reflète le cloisonnement tel qu'il a d'abord été conçu, c'est à dire n'induisant pas une séparation ferme ou absolue entre l'intérieur et l'extérieur.

L'occidentalisation a été accomplie avant que l'on se soit vraiment interrogé sur les

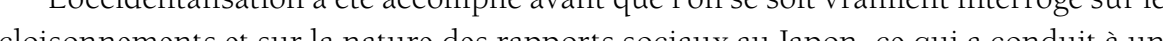
cloisonectife

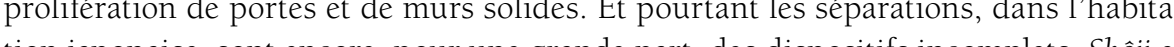

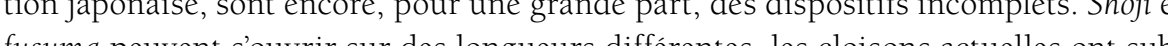
fusuma peuvent sounir sur des longueurs differentes, les cloisons actuelles ont subi bien des transformations, hais elles restent toujours, houvantes et incompletes. Tou aussi persistantes sont les relations sociales, elles-mêmes mouvantes et floues. On serait donc tente d'y voir un trait propre a la culture japonaise. Ainsi, à travers la notion de séparation ou cloisonnement, se pose la question de la sensibilité, de la conscience, et des modes de représentation.

\section{Tsukuroi}

Au Japon, lorsque l'on répare, restaure ou racommode un objet, il n'est pas rare qu’on laisse des traces de cette « amélioration ». Ainsi dans les vieilles constructions, il parait

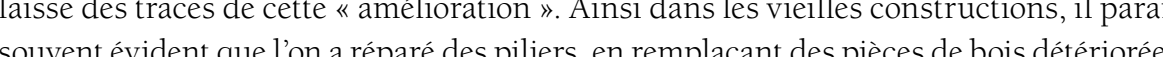
sonve du bois neur. Du point de vue de loccident, ce type de restauration ou de repartion par du bois neuf. peut sembler nettement insuffisant.

La notion de réparation est exprimée par tsukuroi ; tsukurou dans le sens de « améliorer » a une signification tres proche de tsukuru, « faire » ou « fabriquer » une chose. Mais ce terme s'ecrit à laide de deux sinogrammes qui désignent : le fil, ito, et le bien, zen; s'agit donc d'apporter une amélioration à l'aide d'un fil (ou d'un autre element extérieur) Dans la culture japonaise, il est dérangeant, inconvenant (busahô) de laisser en l'éta les shôji dont le papier s'est déchiré, ou les vêtements abîmés. Mais quand ils sont réparés, cela reste visible. Un vêtement troué ou déchiré n'est pas acceptable, mais on le porte san réticence sil a été raccommodé. Au milieu du xxe siècle encore, on pouvait voir des paysans porter des vêtements couverts de " raccomodages ".

De la même manière, dans les habitations, on répare régulièrement le toit de chaume, de tuiles ou de bardeaux, en remplaçant les parties détériorées. Appelée tsukuroi, la réparation des toits en bardeaux se fait en retournant simplement les planchettes de bois sur elles-mêmes. On restaure la base des piliers des maisons et des temples en renouvelant - de façon bien évidente - les parties dégradées par la pluie et les insectes. Le papier épais des fusuma et surtout le papier fin et translucide des shôji, qui subissent souvent des dommages, sont réparés par un simple collage de morceaux de papier sur les parties trouées.

Quant à la réparation des objets en porcelaine ou céramique, elle peut se faire à l'aide d'agrafes, par recuisson après ajout de glaçure sur la fềlure (méthode yaki-tsugi), par une application de laque et de poudre d’or (méthode dite kin-tsugi, considérée comme la plus élaborée)

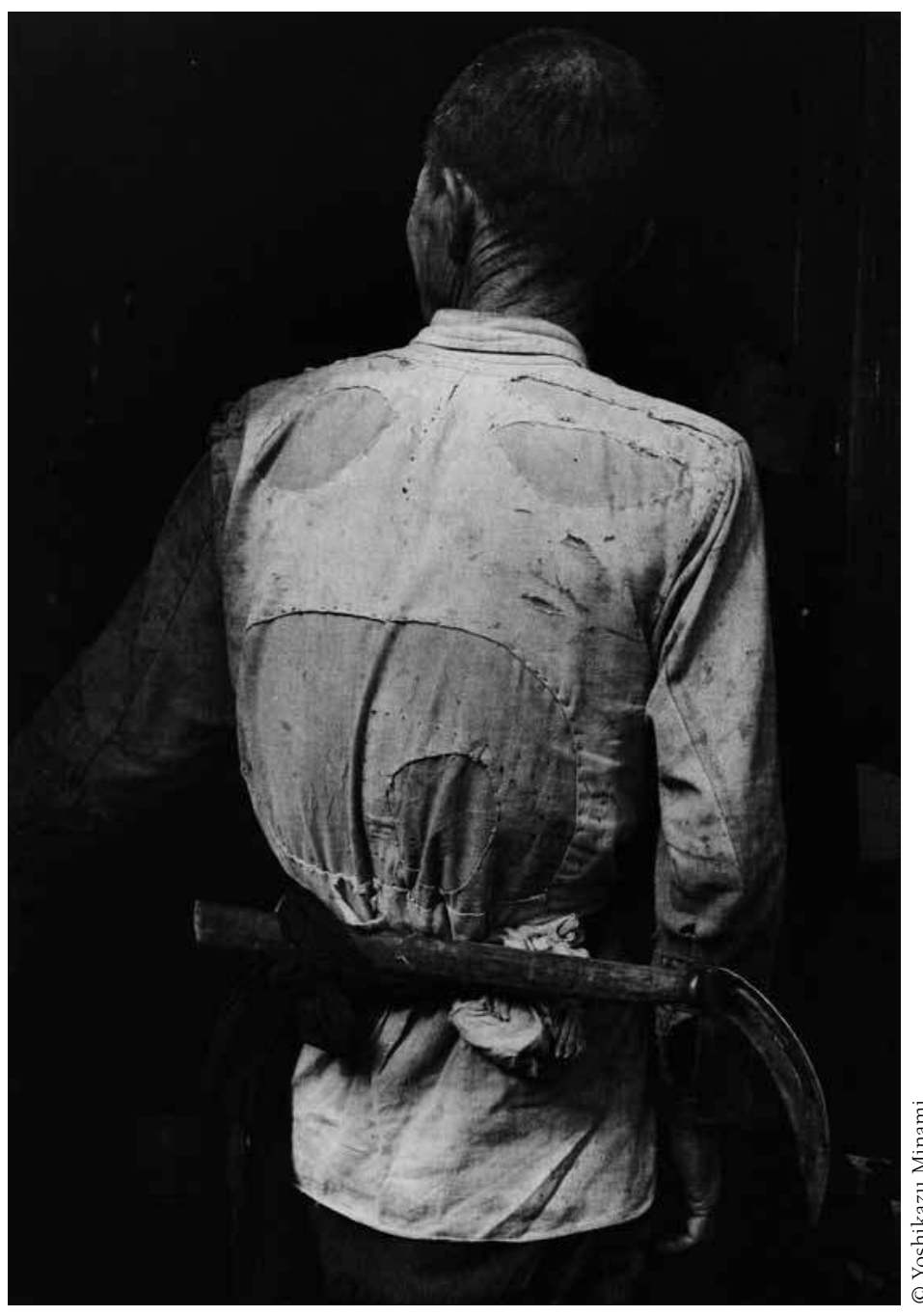

Vetement paysan

Dos couvert de rapiéçages,
milieu du xxes siècle. 


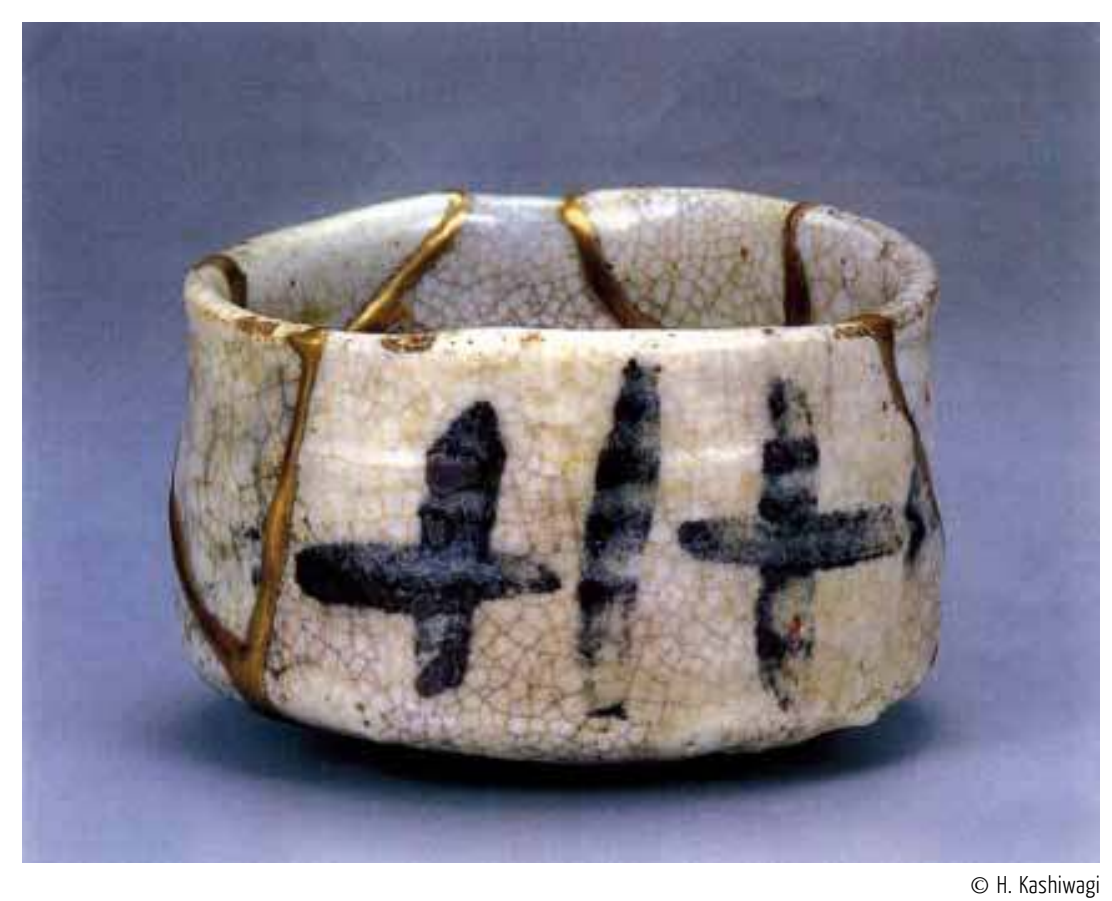

ou par ajout de morceaux issus d'autres pièces, en remplacement des morceaux manquants de l'objet cassé (méthode connue sous le nom de yobi-tsugi, « pièce rapportée »). Alors qu'en occident on cherche à dissimuler les réparations, et que le recollage, our être réussi, ne doit pas laisser de traces, au Japon c'est la réparation-même qui pour êre rensi, ne dit précie tout particulièrement un raccommodage ou une qui tion visibles, et de bonne facture.

Il s'ensuit que les vieux objets réparés sont acceptés comme s'ils navaient jamais été abîmés, comme quelque chose qui naa jamais été dégradé. Il semble même que l'on apprécie particulièrement la beauté de leurs réparations.

Les réparations étant presque toujours mises en évidence, on peut en faire l'éloge, admirer la performance technique, mais aussi vanter leur qualité esthétique, leur beauté intrinsèque. En outre on apprécie les efforts qui ont été faits pour réussir ces restaurations, et même on aime y voir jusqu'a lattachement que l'on éprouve pour l'objet qui a été abîmé, et que ses réparations manifestent.
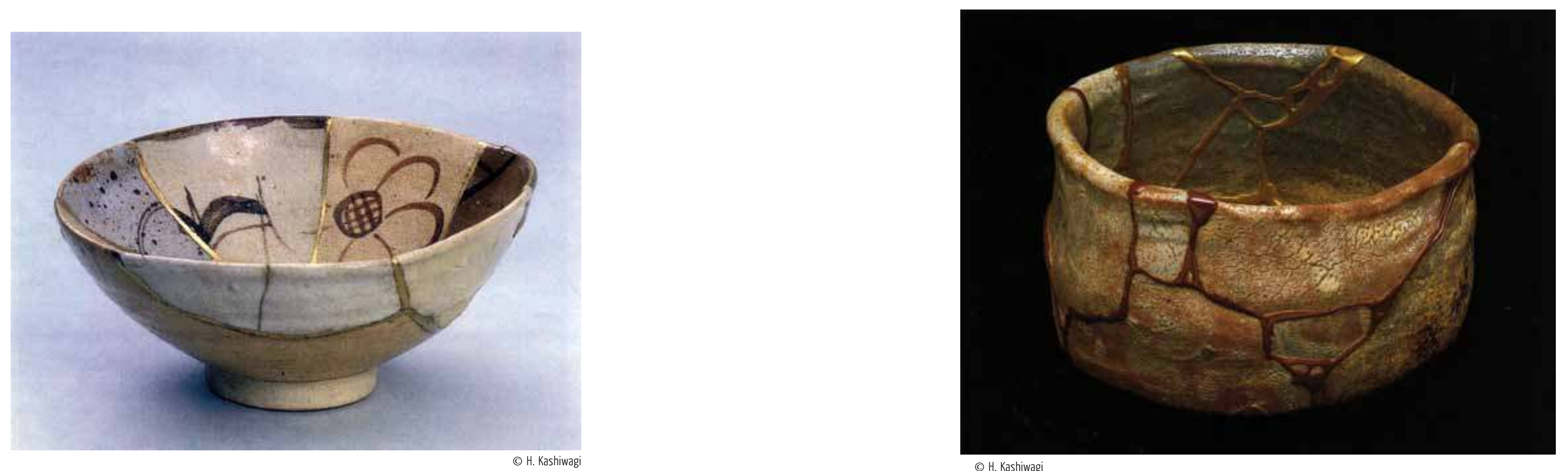

Bol de style Shino, connu sous époque Momovamana, xve-xer siècle

Répré é selon le mode dit yobitsugi (" pièce repportée «): : des morceaul manquants de l'objet cassé sont 


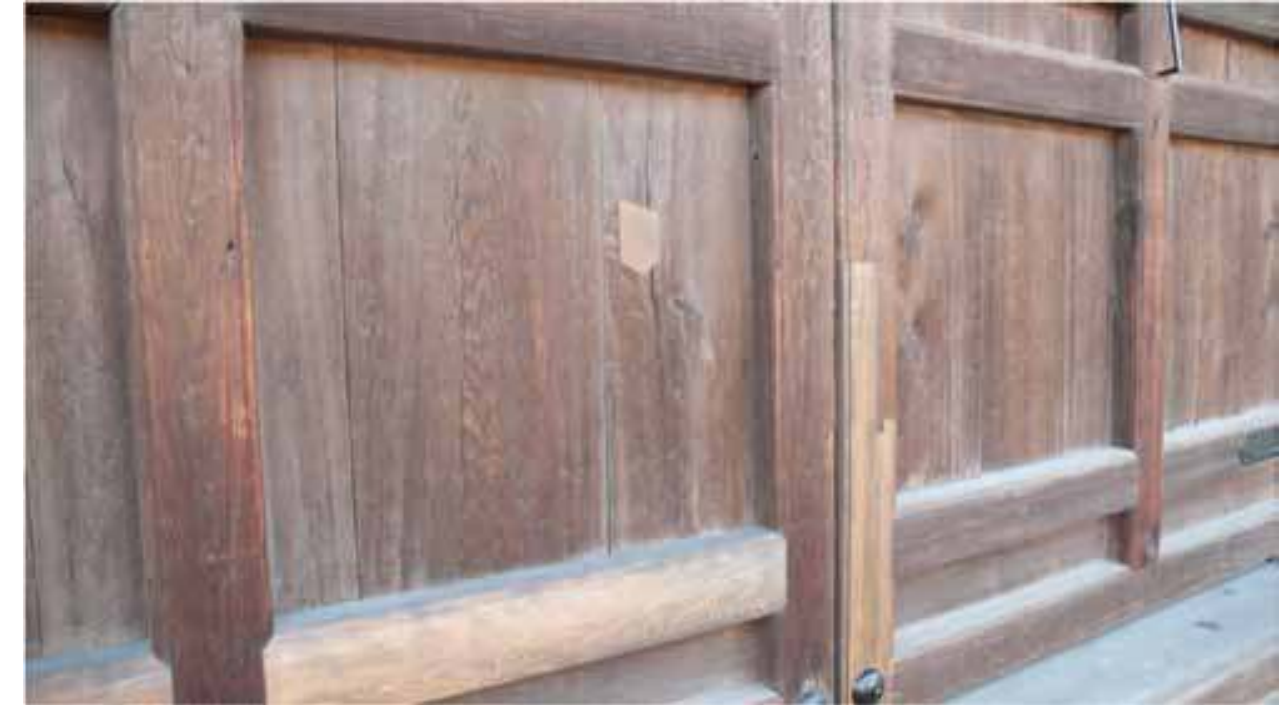

๑ H. Kashiwag

Prendre en charge un objet et s'en occuper au point de le réparer (tsukuro-u) quand il est abîmé, c'est être sensible à la vie de cet objet, faire preuve d'un respect, d'un certain

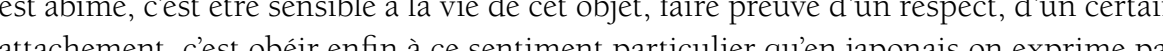
mono no aware (u la tendresse rou a la poignance on des choses). I'objet et le sente par d'aware quil prove se retrouve ainsi dans un univers sensible, le comportement de type émotionnel manifestant la symbiose avec cet objet.

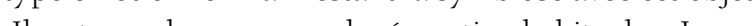

II reste que le processus de réparation habituel au Japon, vu de l'extérieur, a l'air bien incomplet ; il évoque lui aussi, sur un autre mode, l'idée d'inachevé déjà rencontrée propos du cloisonnement.

\section{$\&$}

En conclusion, on retiendra que ces deux notions, séparation et réparation, ont en commun d'évoquer une singularité de la culture japonaise, l'accent mis volontiers sur l'inachèvement ou l'incomplet, ou pour le dire simplement, le goût pour l'imperfection.

Des exemples pris dans différents domaines ont permis d'éclairer ces deux notion qui n'ont pas entre elles de relation directe, mais qui renvoient toutes deux à cette idée d'imperfection

A propos de l'habitation nous avons vu que la notion de cloisonnement est très souple, qu'elle n'implique pas la présence de murs solides comme en occident, et qu'elle n’entraîne pas une distinction nette entrel l'intérieur et l'extérieur. Nous y avons vu aussi un reflet de la souplesse des rapports sociaux. La porosité des cloisons mobiles, qu laissent passer la lumière et les sons, implique un sentiment de continuité entre les deux côtés de la cloison, et en même temps une conscience réciproque de la distinction entre soi et autrui. Mais il est possible que cette notion traditionnelle de séparation se perde un peu, et que s'amenuise aussi le coté souple de nos rapports sociaux, plutôt fondés sur une compréhension ou une adhésion mutuelles.

Nous avons pu voir que cette notion de séparation a un caractère incomplet, qui évoque l'idée d'imperfection, récurrente dans plusieurs domaines de la culture japonaise.

C'est encore à l'idée d'imperfection que renvoie la notion de réparation, que nous avons examinée aussi. Loin de l'idée de restauration invisible recherchée en occident, le mode de réparation traditionnel au Japon illustre une volonté de montrer le procédé ou la trace, et induit un sentiment de proximité avec l'obje réparé, tradrisunt même un atachement, un attendrissement à l'égard de l'obje et de ses blessures.

On peut y voir une particularité de la sensibilité japonaise, en même temps que la prédominance du goût de l'imperfection, si valorisé dans la culture du Japon

\section{Traduit du japonais par Jane Cobbi}




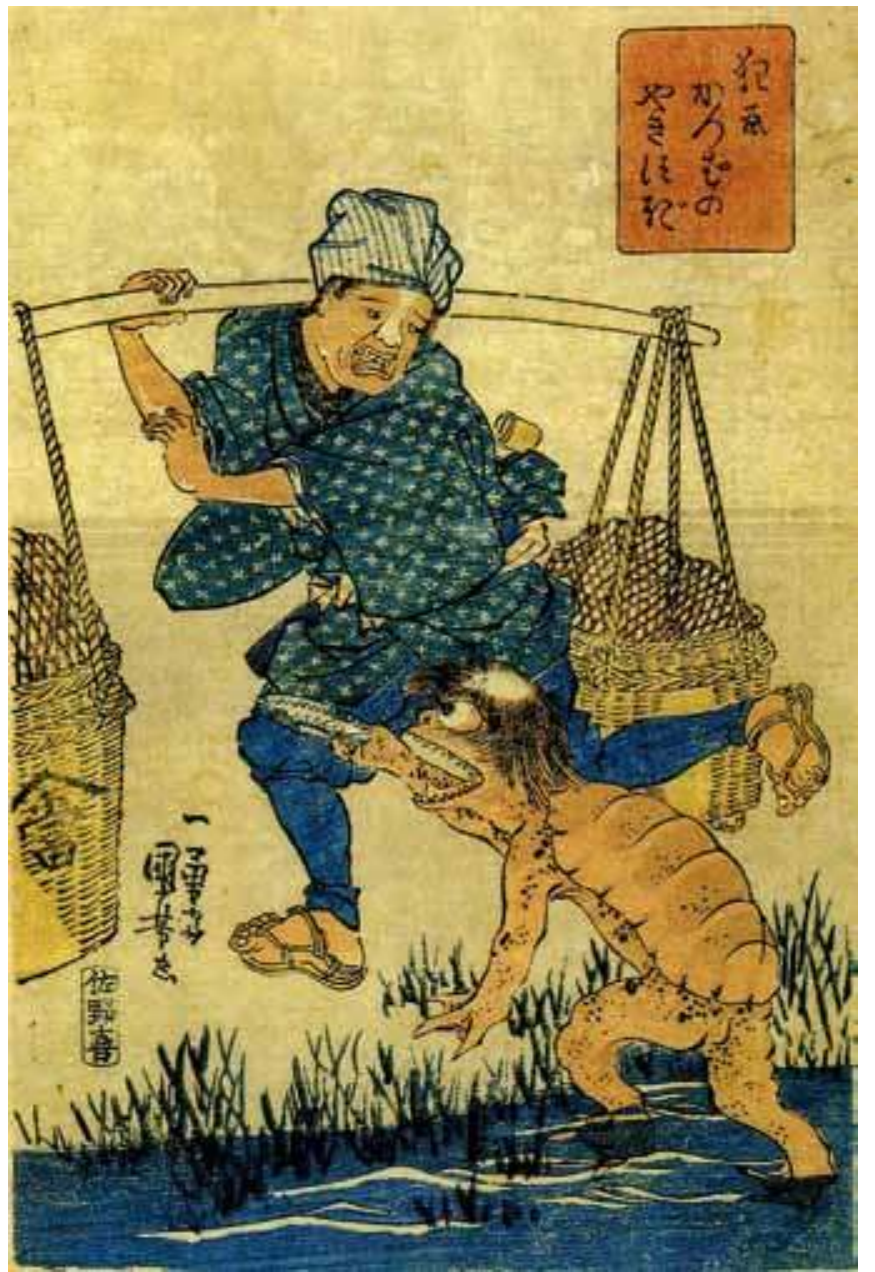

Estampe humoristique de Utagawa
Kuniyoshi, fin de l'époque d'Edo

Uuniyoshi, fin de l'époque d'Edo

de porcelaine et lui demande de

réparer l'assiette de sa tête.

Les kappa sont des créatures

(n)

hivères: on les représentait zvec

au sommet du crâne.

\section{RÉSUMÉ}

Séparation et réparation : expression spatial de l'inachevé au Japon. Dans les représentations de l'espace (ouvert/fermé), du temps (festif/quotidien), et des relations sociales (sphère publique/sphère privée), le Japon a recours a une notion singuliere du cloisonnement ou de la separation, exprimee par shikin.

Dans runivers du monde nateriel, le respect da aux objets usuels (ceramiques, bois, textiles...) - comme aux ceuvres d'art - entraine geneneralement la nécessité de les réparer. Reparation, restauration ou raccommodage, tsu-

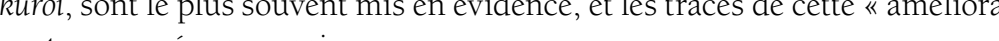
sont conservees avec soin.

Ces deux nas a séparation (shikiri) et réparation (tsukuroi) n’ont pas de relation directe, mais permettent de saisir une conception propre à la culture japonaise, dans laquelle l'inachèvement, l'incomplet ou

\section{ABSTRACT}

Separation and restoration: spatial expression of uncompleteness in Japan. In space (open/close) representations, time (celebration/everyday life) representations or social relation (public sphere/private sphere) representations, Japanese people use an original notion, expressing cloisonning or separation and designated as shikiri.

In material world, attention due to usual objects (potteries, wood objects, clothes, ...) as art pieces does involve the necessity to be repaired. Fixing, restoration or mending, tsukuroi, are made visibles, and the traces of improving is considered as inevitable, and carefully kept.

Those two notions of separation (shikiri) and repair (tsukuroi) have no direct relationship, but allow us to grab a japanese culture conception by which, incompletion, incompleteness or imperfection are highly valuated

\section{MOTS CLÉS}

Espace, temps, inachevé, imparfait, séparation, réparation, rapports sociaux, culture japonaise

\section{KEYWORDS}

Space, time, uncompleted, imperfection, separation, restoration, social relations, japanese culture
Photo d'ouverture : Habitation de fonction (jinya) du début du xixe siècle, réservée aux administrateurs de Takayama, préfecture de Gifu, au centre du Japon. Une pièce recouverte de tatamis donne sur le jardin, par la galerie-engawa.

\section{REEFERENCES}

Ariès, P. 1980 L'Enfant et la vie familiale sous l'Ancien Régime. Paris : Plon. (Pour la traduction japonaise : Ariès, P. 1980 Kodomo no tanjô. Tokyo : Misuzu.).

Kon, W. 1958 Les Variatons de l'habitation (Jûkyo no hensen). In Nihon Minzokugaku taikei 6. Tokyo : Heibonsha, 3-20, 